\title{
A histochemical study of inflammatory lesions of the maxillary sinus mucosa using biotinylated lectins
}

\author{
Takahiro Kaneko, Kazuo Komiyama ${ }^{\dagger}$, Norio Horie, Matsumi Tsuchiya ${ }^{\dagger}$, \\ Itaru Moro ${ }^{\dagger}$ and Tetsuo Shimoyama \\ Department of Oral and Maxillofacial Surgery, Saitama Medical Center, \\ Saitama Medical School, Saitama 350-8550 \\ ${ }^{\dagger}$ Department of Pathology, Nihon University School of Dentistry, Tokyo 101-8310
}

(Received 14 January and accepted 1 June 2000)

\begin{abstract}
The distribution of glycoconjugates in normal maxillary sinus tissues, in cases of maxillary sinusitis and in postoperative maxillary cysts (POMC), was examined using seven different lectins as probes. The results showed that wheatgerm agglutinin (WGA), peanut agglutinin (PNA), Ulex europaeus agglutininI (UEA-1), Ricinus communis agglutinin-I (RCA-l), and concanavalin $A(C o n A)$ strongly react with the cilia and goblet cells. The binding of WGA, PNA, UEA-1, and RCA-1 was increased in maxillary sinusitis and POMC compared with normal maxillary sinus epithelium, whereas that of ConA was decreased. The decreased binding of ConA suggested that there were fewer mannoside residues in the maxillary sinus epithelium in the inflammatory lesion. The PNA bound to the cilia, goblet cells and mucous glandular cells in maxillary sinusitis and POMC, but not in normal, uninflamed cells, indicating that $D$-galactose was produced by the inflammatory condition. Similar binding patterns of PNA and RCA-1 were found in the cilia and on the surface of the epithelium and in the goblet cells. It is assumed that the carbohydrate moiety in the sinus mucosa is altered in inflammatory conditions. (J. Oral Sci. 42, 87-91, 2000)
\end{abstract}

Key words: glycoconjugate; lectin; maxillary sinus; inflammation; histochemistry

Correspondence to Dr. Takahiro Kaneko, Department of Oral and Maxillofacial Surgery, Saitama Medical Center, Saitama Medical School, 1981, Kamoda, Kawagoe, Saitama 350-8550, Japan

\section{Introduction}

Many glycoconjugates are present in the body as components of cell membranes, mucus, body fluids, hormones, enzymes, anti-enzymes, connective tissues, immunoglobulins and human blood groups substances, and play an important role in vivo. Previous studies have reported that alterations of the glycoconjugate moiety at the cell surface are correlated with cell differentiation, neoplastic transformation and changes in cell function (13 ). On the mucosal surface, glycoconjugates form a mucous barrier, which is important for mucosal defense against various pathogenic invaders. Glycoconjugate composition and the amount of mucous secretion may be different in various physiological and pathological conditions.

In the present study, we performed a histochemical analysis of lectin binding in the maxillary sinus mucosa, under normal conditions, in cases of maxillary sinusitis and in postoperative maxillary cysts (POMC), among patients attending the Department of Oral and Maxillofacial Surgery. We compared the lectin binding patterns in the maxillary sinus mucosa between normal and inflammatory disease conditions.

\section{Tissue samples}

The sinus mucosal tissues were obtained from files of the Department of Pathology, Nihon University School of Dentistry, and the Tobu Regional Hospital, Tokyo, between 1996 and 1997. The 22 patients had been diagnosed as having maxillary sinusitis (11 cases) and POMC (11 cases) and had undergone a radical surgery of the maxillary antrum. For the controls, we used 5 cases of histopathologically normal tissues that were taken from non- 
inflammatory sites in patients with maxillary sinus carcinoma.

\section{Lectin and histochemical staining}

Seven kinds of biotinylated lectins were used in this study: concanavalin A (ConA), wheatgerm agglutinin (WGA), Ricinus communis agglutinin-1 (RCA-1), Ulex europaeus agglutinin-1 (UEA-1), peanut agglutinin (PNA), soy bean agglutinin (SBA) and Dolichos biflorus agglutinin (DBA), all purchased from Vector Laboratories Inc. (Table 1).

All tissues were fixed in $10 \%$ neutral buffered formalin and embedded in paraffin by routine procedures. Serial sections were cut at a thickness of $6 \mu \mathrm{m}$, deparaffinized in xylene and cleaned with gradient ethanol. Endogenous peroxidase activity was blocked with methanol containing $0.3 \% \mathrm{H}_{2} \mathrm{O}_{2}$ for $20 \mathrm{~min}$, then washed in phosphate-buffered saline (PBS, pH 7.2). To reduce non-specific staining, the sections were incubated with $10 \%$ bovine serum albumin in PBS for $15 \mathrm{~min}$. The sections were incubated for $60 \mathrm{~min}$ with an appropriately diluted biotinylated lectin at room temperature, then washed 3 times in PBS. Streptavidin horseradish peroxidase complex was added for $30 \mathrm{~min}$ (Zymed, San Francisco, CA, USA). The sections were incubated with a solution containing 3,3'-diaminobenzidine tetrahydrochloride and $\mathrm{H}_{2} \mathrm{O}_{2}$ for 10 min. Finally, the sections were counterstained with Mayer's hematoxylin solution, washed, dehydrated and mounted. For the control staining, we used PBS instead of the biotinylated lectins, or preincubated the specific glycosides to show that the specific lectin bindings had been performed.

\section{Normal sinus tissue}

\section{Results}

The maxillary sinus epithelium was composed of ciliated columnar epithelium in the surface layer and cuboidal epithelial cells in the basal layer, intermingled with goblet cells (Fig. 1a). The lectin binding pattern of normal epithelium showed that ConA reacted with all epithelial layers, with strong staining in the cilia. WGA showed strong positive staining in the cilia and positive staining in goblet cells and mucous glandular cells of the submucosa. RCA-1 showed positive staining in the cilia, while other parts were weakly positive or negative. UEA-1 showed positive staining in all epithelial cells and also in the blood capillary and mucous glandular cells of the submucosa. The sections exposed to PNA revealed almost no staining, while weakly positive staining was seen in the granules of the supranuclear area in the epithelial cells in 2 cases. Other lectins, SBA and DBA, did not stain the sinus mucosa except for weak positive stainig in the granules of the supranuclear area (Table 2).

Table 1. Biotinylated lectin used in this study and thier sugar specifisities

\begin{tabular}{lccc}
\hline \hline \multicolumn{1}{c}{ Lectin } & Abbreviation & Dilution & Sugar specificities \\
\hline Concanavalin A & ConA & $1 / 200$ & D-mannose \\
Wheat germ agglutinin & WGA & $1 / 200$ & N-acetyl-D-glucosamine \\
& & & N-acetyl-D-neuraminic acid \\
Ricinus communis agglutinin 1 & RCA-1 & $1 / 200$ & D-galactose \\
Ulex europaeus agglutinin 1 & UEA-1 & $1 / 200$ & $\alpha$-L-fucose \\
Peanut agglutinin & PNA & $1 / 100$ & $\beta$-D-galactose \\
& & & $\beta$-N-acetyl-D-galactosamine \\
Soy bean agglutinin & SBA & $1 / 100$ & N-acetyl-D-galactosamine \\
Dolichos biflorus agglutinin & DBA & $1 / 100$ & $\alpha$-N-acetyl-D-galactosamine \\
\hline
\end{tabular}

Table 2. Lectin binding of sinus mucosa

\begin{tabular}{|c|c|c|c|c|c|c|c|c|c|c|c|c|c|c|}
\hline & \multicolumn{2}{|c|}{ Con $\mathrm{A}$} & \multicolumn{2}{|c|}{ WGA } & \multicolumn{2}{|c|}{ RCA- 1} & \multicolumn{2}{|c|}{ UEA-1 } & \multicolumn{2}{|c|}{ PNA } & \multicolumn{2}{|c|}{$\mathrm{SBA}$} & \multicolumn{2}{|c|}{ DBA } \\
\hline & $\mathrm{N}$ & MS/POMC & $\mathrm{N}$ & $\mathrm{MS} / \mathrm{POMC}$ & $\mathrm{N}$ & MS/POMC & $\mathrm{N}$ & MS/POMC & $\mathrm{N}$ & MS/POMC & $\mathrm{N}$ & MS/POMC & $\mathrm{N}$ & $\overline{M S / P O M C}$ \\
\hline cilia & ++ & ++ & ++ & ++ & + & ++ & + & + & - & \pm & - & - & - & - \\
\hline epithelial cell (surface) & + & + & \pm & \pm & - & \pm & \pm & \pm & \pm & + & $\pm \neq$ & $\pm \neq$ & $\pm \#$ & $\pm \#$ \\
\hline epithelial cell (basal) & + & \pm & \pm & + & - & \pm & \pm & + & - & - & - & - & - & - \\
\hline goblet cell & + & \pm & + & + & \pm & + & \pm & \pm & - & + & - & - & - & - \\
\hline capillary & - & - & - & - & - & - & + & + & - & - & - & - & - & - \\
\hline mucous gland & + & + & + & + & \pm & + & + & + & - & \pm & - & - & - & - \\
\hline $\begin{array}{l}\mathrm{N} \text { : normal sinus } \mathrm{m} \\
\mathrm{MS} / \mathrm{POMC} \text { : maxil }\end{array}$ & $\begin{array}{l}\operatorname{cosa}( \\
\text { ry } \sin \end{array}$ & $\begin{array}{l}n=5) \\
\text { usitis }(n=\end{array}$ & 11) & post o & . & maxil & & 11) & + & $\begin{array}{l}\text { negative } \\
\text { positive }\end{array}$ & & $\begin{array}{r} \pm: \\
++:\end{array}$ & $\begin{array}{l}\text { veak } \\
\text { trong }\end{array}$ & \\
\hline
\end{tabular}

\#: granules of epithelial cells 


\section{Maxillary sinusitis and POMC}

The maxillary sinusitis and POMC specimens were histologically characterized by hyperplasia of the sinus mucosa with an irregular lining of epithelium. Pseudostratified epithelial hyperplasia of the epithelial lining was often identified (Fig.1b). Both maxillary sinusitis and POMC lesions showed similar histological features. Thus, the difference between the lesions was only the degree of inflammatory change, represented by the number of infiltrating cells and the amount of fibrous tissue and hyalinization found in the submucosa. In both inflammatory lesions, augmented mucus secretion was found because the number of goblet cells and mucous glands were increased. The numbers of capillaries were also increased in both lesions.

The lectin binding patterns in maxillary sinusitis and POMC revealed some differences from normal mucosa (Table 2). Although the ConA staining patterns were similar, the staining intensity in goblet cells and the basal cell layer of epithelium was decreased in the lesions (Fig. 2a). The sections incubated with WGA showed an increase of staining intensity in the epithelial basal cell layer of maxillary sinusitis and POMC (Fig. 2b). The sections incubated with RCA-1 showed an increase of staining intensity in the cilia, goblet cells and mucous glands, and the staining pattern was turned to positive in the surface and basal layers of the epithelium (Fig. 2c). UEA-1 showed a staining intensity similar to that of normal mucosa except for an increase of staining in the basal cell layer of the epithelium (Fig. 2d).

In the PNA sections, the number of positively stained granules in the supranuclear area of the epithelium was clearly increased in maxillary sinusitis and POMC. In addition, the cilia, goblet cells and submucosal glands of maxillary sinusitis and POMC were positively stained by PNA, while these were negative in the normal mucosa. Thus, PNA revealed staining patterns that differed between normal and inflammatory conditions (Fig. 2 e, f). SBA and DBA were negative except for in the granules of the surface layer.

\section{Discussion}

The glycoconjugates in the nasal glands and maxillary sinus are found not only in the sugar coating of the cell surface and intercellular matrix, but also in the mucous which play an essential role in the normal function of muco-ciliary clearance (4). It is suspected that glycoconjugate alterations are correlated with the transformation of chronic maxillary sinusitis with hyperplasia of the sinus mucosa and hypersecretion.

The benefit of using the lectin binding method for glycoconjugate analysis is that it specifically identifies the carbohydrate residues in cells and tissues. Sialomucin and sulfomucin can be detected by alcian blue and high-iron diamine. However, these methods cannot distinguish carbohydrate composition or particular carbohydrate residues. The carbohydrate moieties of cell surfaces are involved in cell-to-cell interaction and are thought to be important in cell functions, including intercellular recognition and cell maturation $(5,6)$. Alteration of the microenvironment and local inflammatory reactions in a variety of diseases may influence cell surface carbohydrate moieties $(7,8)$.

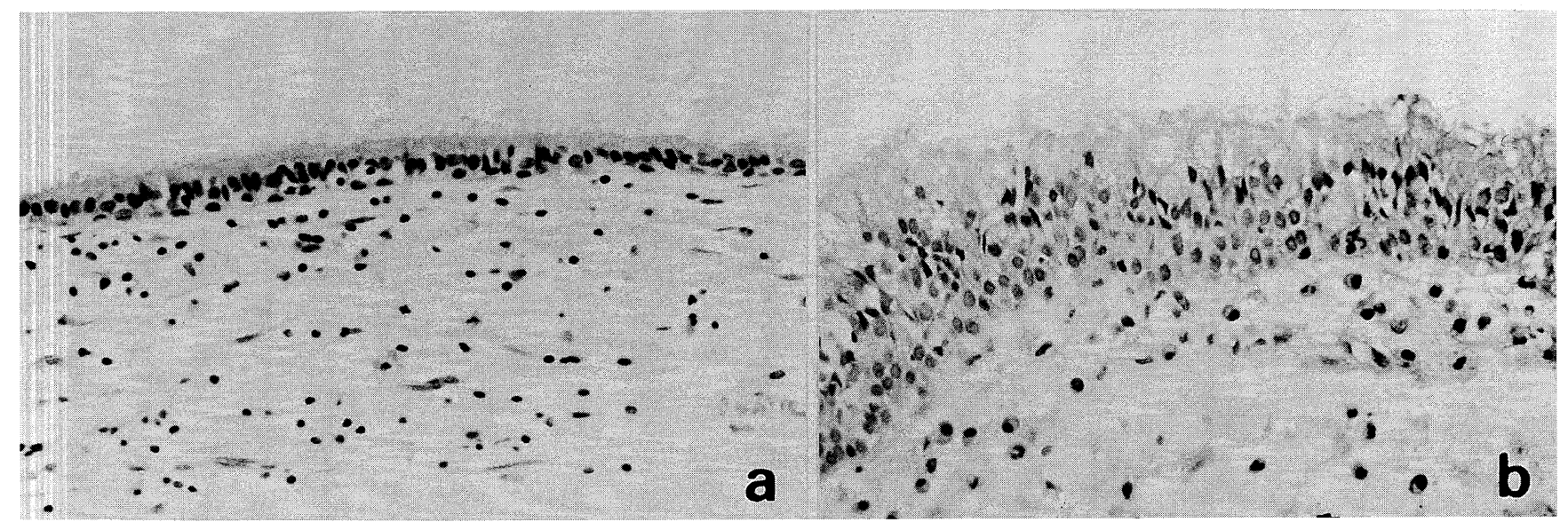

Fig 1. (a) Normal sinus mucosa; maxillary sinus mucosa is covered with ciliated columnar epithelium in the surface layer and cuboidal epithelial cells line the basal layer (HE stain, $\times 200$ ). (b) maxillary sinusitis and POMC; pseudostratified epithelial hyperplasia of the sinus mucosa with irregular lining of the epithelium and increment in the number of goblet cells are observed (HE stain, $\times 400)$. 
The carbohydrates of the cell surface have been revealed to be hetero-oligosaccharides that consist of residues of several sugars, such as hexose, deoxyhexose, hexamine, sialic acid and pentose. Among these monosaccharides, fucose, galactose, sialic acid, $\mathrm{N}$-acetyl-glucosamine, and
$\mathrm{N}$-acetyl-galactosamine have generally been noted in studies of glycoconjugates. Fucose and sialic acid, especially, are important components as they have a tendency to be distributed at the terminal of sugar residues (10).
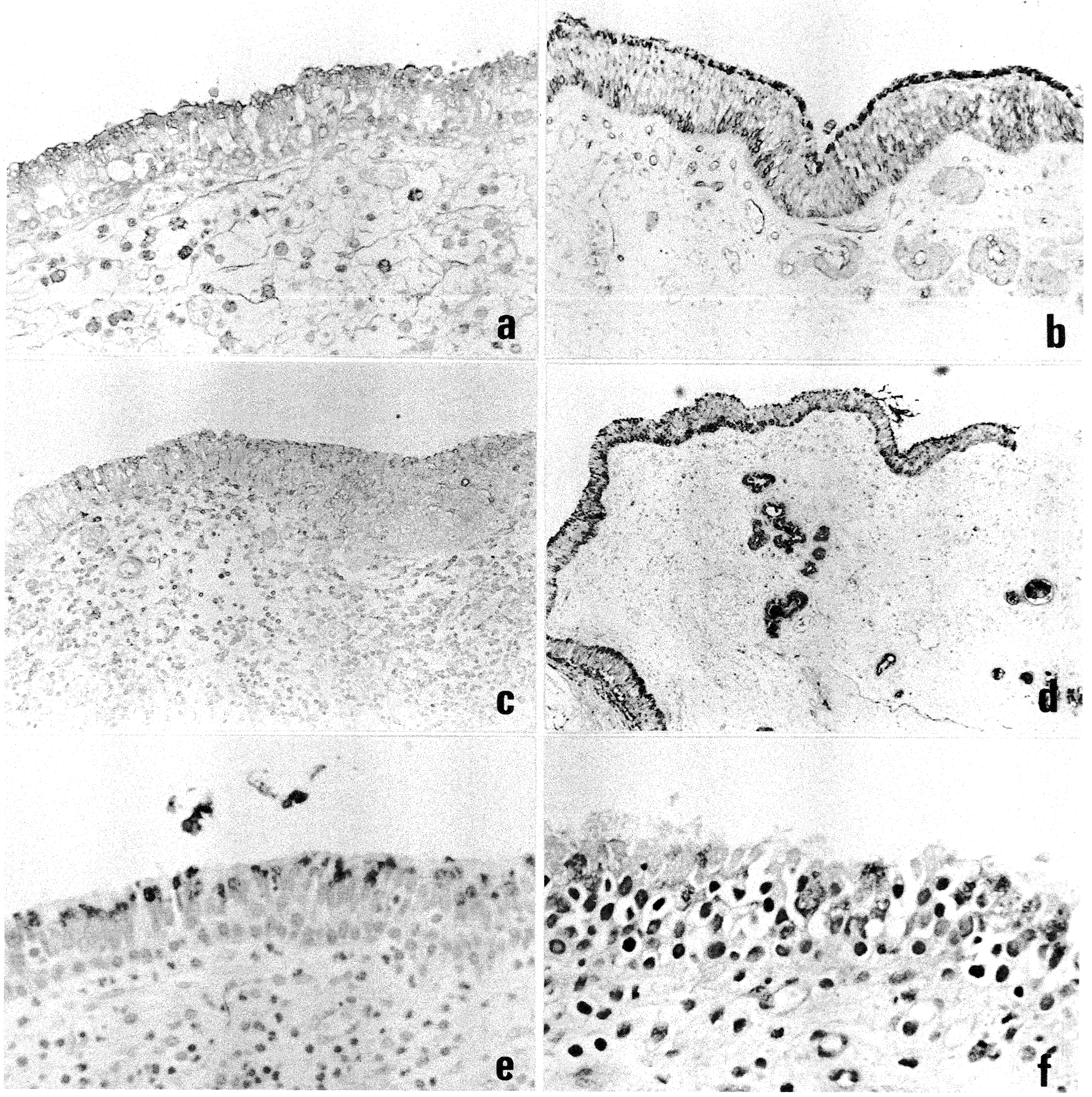

Fig 2. Lectin profiles of inflammatory mucosa. (a) The cilia and surface of the mucous epithelium are positive for ConA (ConA stain, $\times 200$ ). (b) WGA shows strong positive staining of the cilia and basal cell layer (WGA stain, $\times 200$ ). (c) RCA-1 shows moderate staining over all the epithelial layer (RCA-1 stain, $\times 200$ ). (d) The cilia and basal cell layer and the mucus -secreting cells are strongly positive for UEA-1 (UEA-1 stain, $\times 100$ ). (e) PNA shows moderate staining in the supra nuclear region of the epithelial cells (PNA stain, $\times 200$ ). (f) The goblet cells in sinus inflammatory lesions show moderate positive staining for PNA (PNA stain, $\times 400$ ). 
Maxillary sinusitis is caused by various infectious agents, such as bacteria, fungi, and viruses. Otorhinolaryngologists have focused on viral infections and non-bacterial antigens that reach the sinus, whereas oral surgeons have studied bacterial infection through periapical periodontitis. Several studies have reported the presence of glycoconjugates in the respiratory tract and the nasal-sinus region detected by lectins $(9,10)$. However, alteration of glycoconjugates in maxillary sinusitis or POMC has not been extensively studied.

We compared the distribution of glycoconjugates in a normal maxillary sinus and in maxillary sinusitis and POMC using seven different lectins as probes. The results showed that Con A, WGA, RCA-1, UEA-1 and PNA predominantly reacted with the cilia and goblet cells. The staining intensities of WGA, RCA-1, UEA-1 and PNA were increased in inflammatory conditions, whereas that of ConA was decreased. Normal sinus mucosa showed a similar staining pattern, as described previously by Jin et $\mathrm{al}(10)$. It has been reported that changes in glycoconjugates can be found in the sinus epithelium and goblet cells in experimental sinusitis. Enhanced fucosylation and sialation of the mucosa have been observed in the inflammatory process of sinusitis (11).

In this study, we also found an alteration of glycoconjugate expression in the inflamed mucosal epithelium. The numbers of goblet cells and the amount of mucous secretions were increased in maxillary sinusitis and POMC. Similar patterns of increased PNA and RCA-1 staining were found in the cilia and goblet cells. It is assumed that an increased amount of mucus was secreted from the goblet cells which covered the mucosal surface. Decreased ConA binding was also observed in the goblet cells in maxillary sinusitis and POMC compared with normal maxillary sinus tissue. It is suggested that the mannoside residues of the maxillary sinus epithelium were decreased in the inflammatory lesion. PNA also bound with the cilia, goblet cells and mucous glands in maxillary sinusitis and POMC, but not in the normal condition, indicating that $\mathrm{D}$-galactose was present in the goblet cells and mucous glandular cells under inflammatory conditions. The alteration of the carbohydrate moiety and the increased secretions in maxillary sinusitis and POMC reflected the defense mechanism of the maxillary sinus mucosa against inflammatory agents.

\section{Acknowledgments}

We thank Dr. Tadao Okano for providing the control samples for this study.

\section{References}

1. Kannan, S., Balaram, P., Chandran, G. J., Pillai, M. R., Mathew, B. and Nair, M. K. (1993) Expression of lectin-specific cellular glycoconjugates during oral carcinogenesis. J. Cancer Res. Clin. Oncol. 119, 689694

2. Vijayan, K.K., Remani, P., Beevi, V.M., Ankathil, R., Vijayakumar, T., Rajendran, R., Augustine, J. and Vasudevan, D.M. (1987) Tissue binding patterns of lectins in premalignant and malignant lesions of the oral cavity. J. Exp. Pathol. 3, 295-304

3. Saku, T. and Okabe, H. (1989) Differential lectinbindings in normal and precancerous epithelium and squamous cell carcinoma of the oral mucosa. J. Oral Pathol. Med. 18, 438-445

4. Sakakura, Y., Majima, Y., Saida, S., Ukai, K. and Miyoshi, Y. (1985) Reversibility of reduced mucociliary clearance in chronic sinusitis. Clin. Otolaryngol. 10, 79-83

5. Roseman, S. (1970) The synthesis of complex carbohydrates by multiglycosyltransferase systems and their potential function in intercellular adhesion. Chem. Phys. Lipids 5, 270-297

6. Sharon, N. and Lis, H. (1972) Lectins: cellagglutinating and sugar- specific proteins. Science 177, 949-959

7. Zieske, J. D. and Bernstein, I. A. (1982) Modification of cell surface glycoprotein: addition of fucosyl residues during epidermal differentiation. J. Cell Biol. 95, 626-631

8. Brabec, R. K., Peters, B. P., Bernstein, I. A., Gray, R. H. and Goldstein, I. J. (1980) Differential lectin binding to cellular membranes in the epidermis of the newborn rat. Proc. Natl. Acad. Sci. USA. 77, 477479

9. Fukami, K. (1985) Histochemical study of the glycoconjugates in human upper airway mucosa. Nippon Jibiinkoka Gakkai Kaiho 88, 449-454 (in Japanese)

10. Jin, C., Ukai, K., Sakakura, Y., Yamada, H. and Masuda, S. (1989) Electron microscopic study of glycoconjugate in nasal mucosa of nasal allergy by lectin histochemistry. Nippon Jibiinkoka Gakkai Kaiho 92, 709-715 ( in Japanese )

11. Otori, N., Carlsoo, B. and Stierna, P. (1998) Changes in glycoconjugate expression of the sinus mucosa during experimental sinusitis: a lectin histochemical study of the epithelium and goblet cell development. Acta Otolaryngol. 118, 248-256 